\title{
Effects of stimulus spacing on steady state gradients of inhibitory stimulus control
}

\author{
SUSAN M. ESSOCK and DONALD S. BLOUGH \\ Brown University, Providence, Rhode Island 02912
}

\begin{abstract}
When the response of pigeons is maintained to a number of stimulus wavelengths, but extinguished to one (S-), the birds peck more rapidly at stimuli near the $S-$ than at more distant stimuli. The present study explores this "dimensional contrast" effect as a function of the number and spacing of test wavelengths. A fixed portion of the wavelength continuum was spanned by $5,9,13$, or 49 stimuli, which appeared in random sequence behind a standard pecking key. At the end of each 20 -sec trial, pecks to test stimuli produced a conditioned reinforcer (sometimes followed by food), while pecks to the $S$ - stimulus produced only darkness. Dimensional contrast "shoulders" developed to test stimuli on either side of the S-; these shoulders were of approximately the same height and wavelength position for all but the 5stimulus (widely spaced) condition, and were comparable to the original contrast results with 25 stimuli. The results strongly suggest that the extent and locus of contrast shoulders are largely independent of the number and spacing of test stimuli.
\end{abstract}

When a nonreinforced wavelength (S-) is introduced into an otherwise equally reinforced series of randomly presented, closely spaced wavelengths, responding typically decreases at the $\mathrm{S}-$. More unexpectedly, responding has been found to increase in the presence of stimuli near the $S$-, relative to responding to more distant stimuli (Blough, 1975). These peaks of response, or "shoulders," on either side of the S - trough appear at about the same time as response reduction at $\mathrm{S}-$, and they persist through months of training. Blough (1975) has suggested the name "dimensional contrast" for these shoulders and related effects, and he has proposed a model of generalization and discrimination that predicts such effects.

Because of its potential significance to the understanding of discriminative processes, dimensional contrast requires further exploration. One potentially significant variable is stimulus spacing, for contrast shoulders are clearly some function of distance from the S - . But how shall "distance" be interpreted? Blough's model assumes that underlying stimulus similarity is a fixed function of the physical variable (here, wavelength). It predicts that, if shoulders appear, they will form at about the same wavelengths regardless of the number and spacing of the stimuli. An intuitively plausible alternative hypothesis might suggest that contrast shoulders would be determined relative to the particular set of stimuli presented; perhaps responding to whichever stimuli lie relatively

This research was supported in part by USPHS Grant MH02456 to the second author. Reprints may be obtained from Donald S. Blough, Department of Psychology, Brown University, Providence, Rhode Island 02912. close to the $\mathrm{S}$ - will show the contrast effect, regardless of the actual physical distance between the stimuli.

A related point concerns the process by which contrast is produced. The Blough model predicts that shoulders will occur only if stimuli are spaced closely enough that reinforcement of one stimulus affects (via a similarity function) the relative associative strengths of its immediate neighbors. Contrast should disappear in an "easy" discrimination, where the physical distance between stimuli is great enough that the nonreinforcement of S - would not, over a period of time, depress responding at its immediate neighbors and where reinforcement of these neighbors, in turn, would not elevate responding at other stimuli. Alternative hypotheses, some of which have been suggested by Blough (1975), do not require this relatively close spacing of stimuli to produce contrast effects.

The present experiment provides data on the effect of test stimulus spacing upon gradients of stimulus control around $\mathrm{S}$ - stimuli which may contribute to a choice among alternative conceptions of the contrast process.

\section{METHOD}

\section{Subjects}

Three male White Carneaux pigeons served as subjects; all were experimentally naive. They were maintained at $75 \%-80 \%$ of their free-feeding weights by supplementary feedings, when necessary, after experimental sessions.

\section{Apparatus}

The three birds worked simultaneously, each in a standard Lehigh Valley pigeon chamber illuminated by a dim overhead light. Monochromatic stimuli were provided by light from a 
Bausch and Lomb $250-\mathrm{mm}$ grating monochromator, which passed through fiber-optics cables ending behind glass response keys in each box. The stimulus visible through the key was a bright vertical line, $3.5 \times 1.0 \mathrm{~mm}$, on a dark ground. This line assumed a number of different wavelengths during each session, spaced in steps of approximately equal discriminability on either side of $597 \mathrm{~nm}$ (P. M. Blough, 1972; Wright, 1972). A second stimulus pattern-a gray $6 \times 6 \mathrm{~mm}$ diamond on a white ground-replaced the monochromatic line at the end of some trials. The response keys operated on application of about $10 \mathrm{~g}(.1 \mathrm{~N})$ of force. White masking noise was provided in each chamber. A LINC computer controlled the apparatus and recorded the data.

\section{Procedure}

Daily sessions included from 475 to 494 test trials, during which a set of stimulus wavelengths was repeated over and over in varied random orders. In different portions of the experiment, $10,38,53$, or 95 such series were presented, each series including (respectively) $49,13,9$, or 5 wavelengths. A test trial began with the appearance of the stimulus line at a present wavelength. For $20 \mathrm{sec}$, pecks at the key produced no consequences; a maximum of 10 additional seconds was provided, during which the line remained visible until a peck occurred; this peck turned off the line and produced the gray diamond for $3 \mathrm{sec}$. Mixed grain was then offered for a period of about $3 \mathrm{sec}$ with a probability of from $1 / 3$ to $1 / 5$ adjusted according to the grain requirements of the individual subjects. If the bird failed to peck at the stimulus line during the 10 -sec period, the key became dark and a 5 -sec intertrial interval began.

In addition to the test trials just described, most sessions included frequent trials during which the nonreinforced stimulus $(\mathrm{S}-, 597 \mathrm{~nm})$ appeared. These $\mathrm{S}-$ trials were interspersed randomly among the test trials with a probability of $1 / 2$, and they were like the test trials except that the first peck after $20 \mathrm{sec}$ terminated the trial without the appearance of the gray diamond or reinforcement. After an intertrial interval of 5 to $15 \mathrm{sec}$, the next stimulus was presented. The $\mathrm{S}-$ stimulus was also one of the wavelengths used in the baseline procedure first described, so pecking at it could produce the gray diamond stimulus and a possibility of reinforcement $10,38,53$, or 95 times per session, depending upon the number of stimuli used.

Each of the four stimulus spacings was used in blocks of successive daily sessions. During the early sessions with each spacing, $\mathrm{S}$ - trials were omitted (baseline condition) and response rates were permitted to stabilize at a relatively constant level for all test wavelengths. Then sessions including $S$ - were run until stable gradients were achieved. At this point, a new stimulus spacing was introduced and the procedure was repeated, in the order recorded in Table 1. When all four spacings had been tested, each was replicated, this time with a constant number of baseline and $\mathrm{S}-$ sessions in each block (Days 88-167, Table 1).

\section{RESULTS}

The basic pattern of results was similar to that described by Blough (1975). Within each trial, the birds pecked with increasing rapidity. This "scallop" was used to provide a set of four successively higher response baselines, one from each 5 -sec quarter of the interval. The second-quarter rates were chosen as the measure least subject to distortion by lower or upper limit effects.

Figure la shows the mean response rates during the second quarter of the FI for the three birds, at each of the stimulus spacings. Each set of points in Figure la represents the percent of mean total re-
Table 1

Number of Sessions at Each Stimulus Spacing

\begin{tabular}{|c|c|c|c|}
\hline \multirow[b]{2}{*}{ Sessions } & \multicolumn{2}{|c|}{ Number of } & \multirow{2}{*}{$\begin{array}{c}\text { Probability } \\
\text { of S- }\end{array}$} \\
\hline & Days & Stimuli & \\
\hline $1-10$ & 10 & 49 & 0 \\
\hline $11-17$ & 7 & 49 & $1 / 2$ \\
\hline $18-27$ & 10 & 13 & 0 \\
\hline $28-34$ & 7 & 13 & $1 / 2$ \\
\hline $35-45$ & 11 & 5 & 0 \\
\hline $46-57$ & 12 & 5 & $1 / 2$ \\
\hline $58-77$ & 20 & 9 & 0 \\
\hline $78-87$ & 10 & 9 & $1 / 2$ \\
\hline $88-98$ & 11 & 49 & 0 \\
\hline $99-107$ & 9 & 49 & $1 / 2$ \\
\hline $108-118$ & 11 & 9 & 0 \\
\hline $119 \cdot 127$ & 9 & 9 & $1 / 2$ \\
\hline $128-138$ & 11 & 5 & 0 \\
\hline $139-147$ & 9 & 5 & $1 / 2$ \\
\hline $148-158$ & 11 & 13 & 0 \\
\hline $159-167$ & 9 & 13 & $1 / 2$ \\
\hline
\end{tabular}

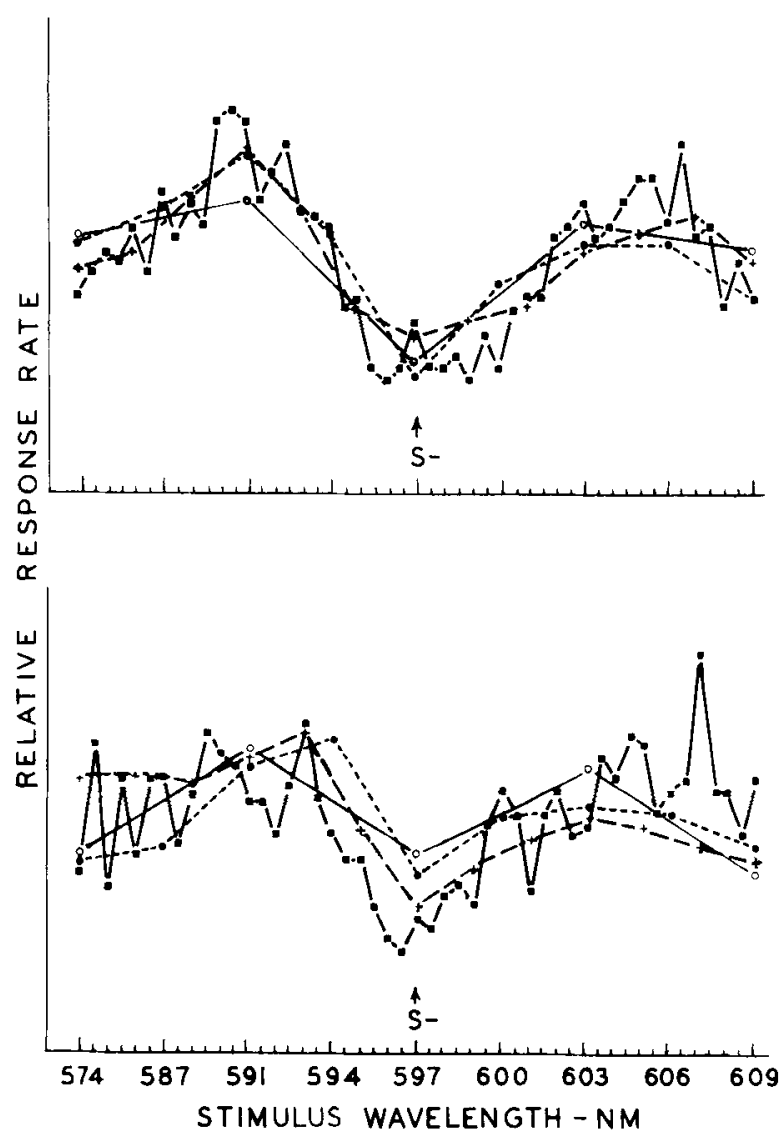

Figure 1. Mean response of three subjects for each stimulus spacing. The data include responses from the second quarter of each trial, averaged over the final six sessions at each spacing. $S$ - appeared with probability of $1 / 2$. To facilitate comparison of curve shapes, the data are plotted as percent of total responding at a given spacing, multiplied by a constant such that the areas under the curves are equal. $O-0,5$ stimuli; $\bullet . . . \bullet, 9$ stimuli; ,+--+ 13 stimuli; $\square-\square, 49$ stimuli. (a) Data from the first set of exposures to each spacing (Sessions 1-87). (b) Data from the second set of exposures (Sessions 88-167). 
sponding for one of the stimulus spacings during the $\mathrm{S}$ - test sessions. Shoulders are evident on both sides of the $\mathrm{S}$ - for all but the 5-stimulus condition, where the effect is minimal if present at all. The shapes of the curves for the 9-, 13-, and 49-stimulus conditions do not appear to differ systematically with respect to the relative height or position of dimensional contrast shoulders on either side of $\mathrm{S}$ - .

The subjects became more variable during the second part of the experiment, during which a fixed number of sessions was run at each spacing (Figure $1 \mathrm{~b}$ ). One subject ceased to show discriminative responding to the $S$ - during the second group of 49 . stimulus sessions (Sessions 99-107), and its data from that period were omitted from Figure 1b. Despite their variability, the data from the remaining subjects suggest that the position of the shoulders had shifted somewhat toward $\mathrm{S}$ - as a result of the additional training.

Figure 2 provides an idea of individual performance and the range of variability in the data. Results appear here for the bird that differed most from mean data on the first set of stimulus exposures. As may be seen, this bird showed rather less consistency in the position of the peak shoulders than is evident in the mean curves, and a somewhat stronger indication of shoulders under the five-stimulus condition.

\section{DISCUSSION}

This study replicates the dimensional contrast shoulders found by Blough (1975) in gradients along a wavelength dimension around an $\mathrm{S}-$. The present study shows the same contrast effect with 9,13 , and 49 stimuli that Blough found with 25 stimuli over a comparable range. These results indicate that the extent and locus of contrast shoulders are largely independent of the number and spacing of test stimuli, and are in accord with the predictions of Blough's model in this respect. The suggestion that the position of the shoulders narrows somewhat with training is also in accord with the model's prediction. However, the data are somewhat unclear on the further prediction that dimensional contrast will

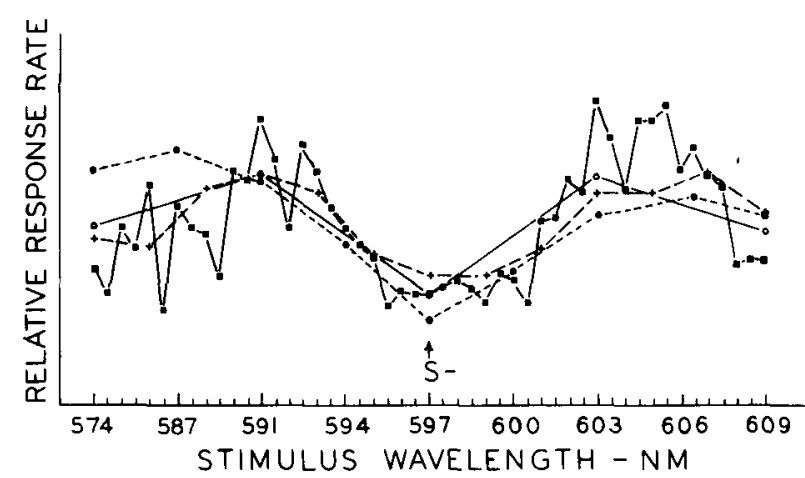

Figure 2. Relative response rates from a single bird. First set of exposures, same conditions and plotting conventions as Figure 1a.

disappear if the test stimuli are sufficiently widely spaced. The 5-stimulus condition was designed to accomplish this; contrast in this condition was indeed minimal on the first determination (Figure 1a), but seemed to reappear to some extent in the replication (Figure 1b). This ambiguity is partly due to variability during the second half of the experiment. In other studies (unpublished), we have often noted similar deterioration of performance in prolonged discrimination tasks. Such deterioration may be related to changes in attention or observing behavior, to the development of competing superstitious responses, or to species-specific behaviors that frequently accompany signals for reinforcement.

\section{REFERENCES}

Blough, D. S. Steady-state data and a quantitative model of operant generalization and discrimination. Journal of Experimental Psychology: Animal Behavior Processes, 1975, 104, 3-21.

Blough, P. M. Wavelength generalization and discrimination in the pigeon. Perception \& Psychophysics. 1972, 12, 342-348.

Wright, A. A. Psychometric and psychophysical hue discrimination functions for the pigeon. Vision Research, 1972, 12, 1447-1464.

(Received for publication July 16, 1976; revision accepted January $24,1977$. ) 